\title{
Diagnostics of low-pressure capacitively coupled RF discharge argon
}

\section{plasma}

\author{
Ali A-K. Hussain ${ }^{1}$, Kadhim A. Aadim ${ }^{1}$, Waleed I. Yaseen ${ }^{2}$ \\ ${ }^{1}$ Department of physics, Collage of Science, University of Baghdad \\ ${ }^{2}$ Department of Astronomy, Collage of Science, University of Baghdad \\ E-mail:kadhim_adem@yahoo.com
}

\begin{abstract}
Low-pressure capacitively coupled RF discharge Ar plasma has been studied using Langmuir probe. The electron temperature, electron density and Debay length were calculated under different pressures and electrode gap. In this work the RF Langmuir probe is designed using $4 \mathrm{MHz}$ filter as compensation circuit and I-V probe characteristic have been investigated. The pressure varied from 0.07 mbar to 0.1 mbar while electrode gap varied from $2-5 \mathrm{~cm}$. The plasma was generated using power supply at $4 \mathrm{MHz}$ frequency with power $300 \mathrm{~W}$. The flowmeter is used to control Argon gas flow in the range of 600 standard cubic centimeters per minute $(\mathrm{sccm})$. The electron temperature drops slowly with pressure and it's gradually decreased when expanding the electrode gap. As the gas pressure increases, the plasma density rises slightly at low gas pressure while it drops little at higher gas pressure. The electron density decreases rapidly with expand distances between electrodes.
\end{abstract} Key words

$R F$ discharge, Langmuir probe, Argon Plasma.

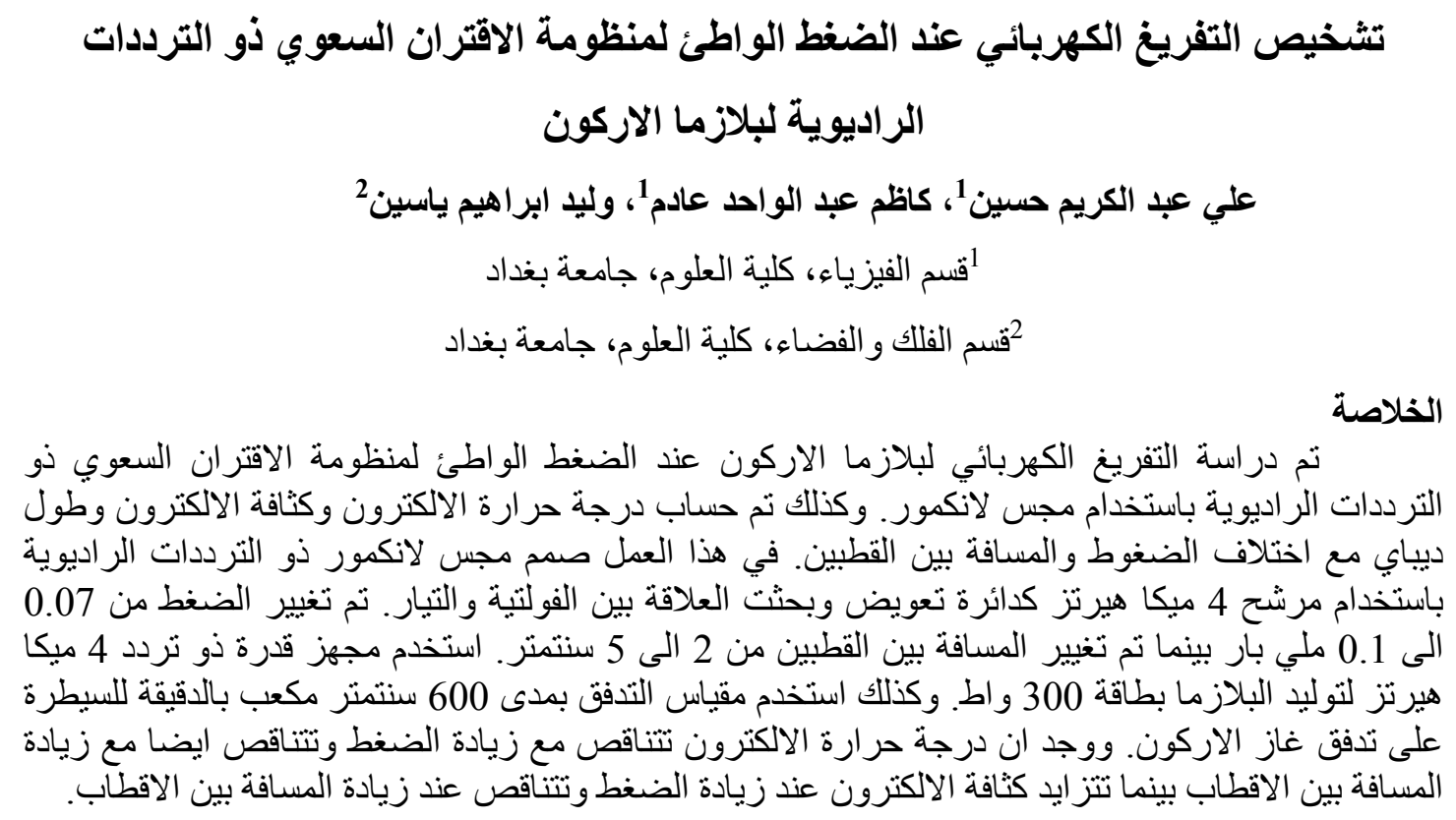

\section{Introduction}

Plasma processing is an important branch of industrial microfabrication and in particular reactive ion etching is generally used for resist etching [1]. Formation of passivation layer, or other plasma technologies applied to the production of integrated 
circuits. From the viewpoints of both industry and physics, experimental and theoretical investigations of $\mathrm{Ar}$ are necessary [2]. A Langmuir probe is a diagnostic instrument that is used to measure electron temperature, and electron density. It consists of single wire inserted into the plasma and measuring the current applied to it at various applied voltages. However, it is an intrusive, not remote, technique; and the wire must be carefully designed so as not to interfere with the plasma nor be destroyed by it [3]. Discharge is produced by applying high voltage RF source of frequency (4 $\mathrm{MHz}$ ) on two Parallel plate systems as shown in Fig. 1.

RF Power 4MHZ

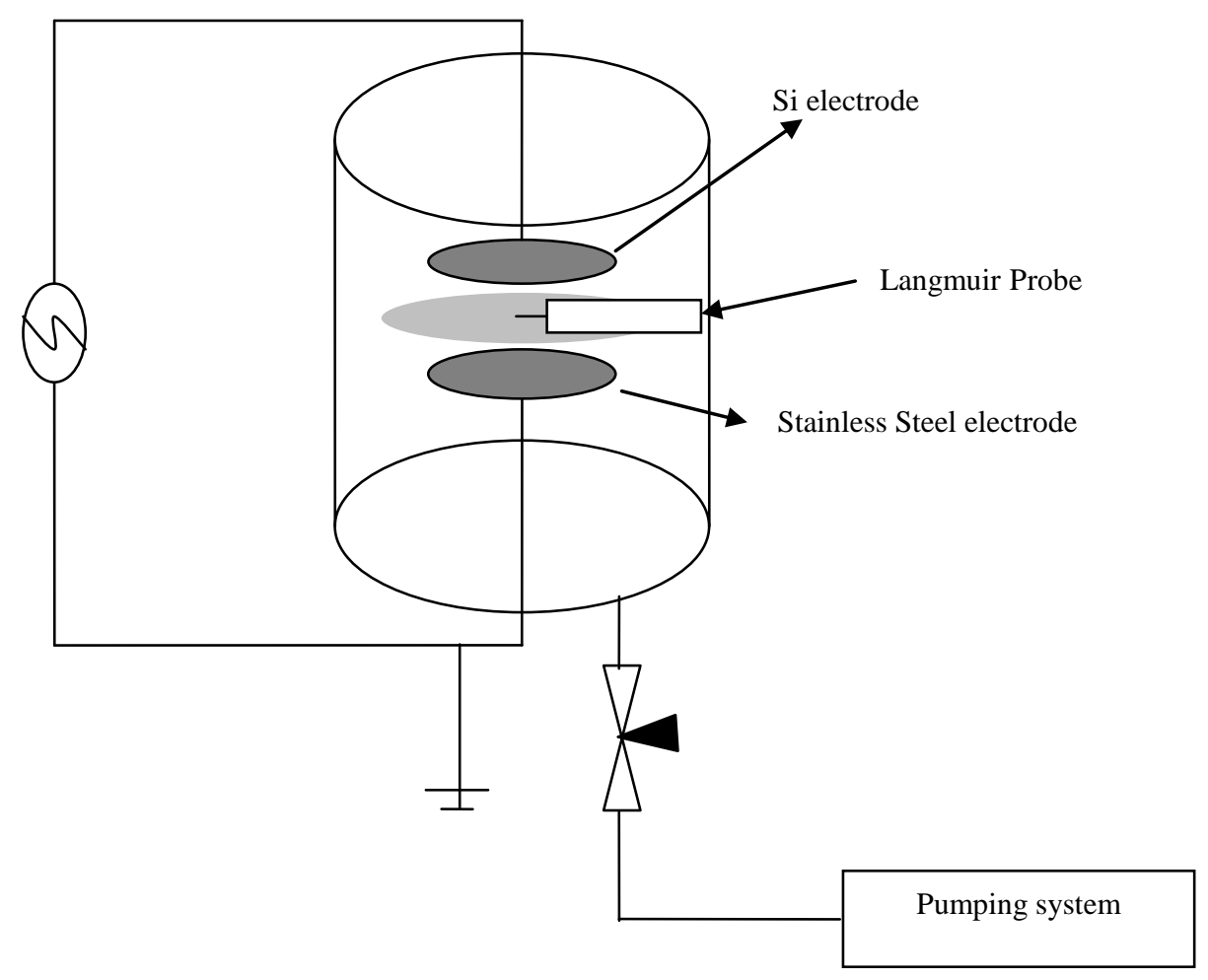

Fig.1: Schematic diagram of the experimental setup.

\section{Probe construction}

A compensated Langmuir probe is used to obtain the I-V characteristic[4]. The probe tip is made of a tungsten wire. The compensation circuit is achieved by winding 15 turns of tungsten wire of the same diameter around the probe; an RF tunable choke is connected just outside the probe and is calculated to be resonant at the fundamental RF discharge frequency. The values of the inductor $\mathrm{L}$ and capacitor $\mathrm{C}$ were obtained from equation:

$f=\frac{1}{2 \pi \sqrt{L C}}$

where $\mathrm{f}$ is the applied RF frequency 4 MHz. The 15 turns winding which represents an external floating electrode is connected to the probe via a $5 \mathrm{nF}$ ceramic capacitor, this connection is housed in the probe ceramic insulator as shown in Fig.2. 


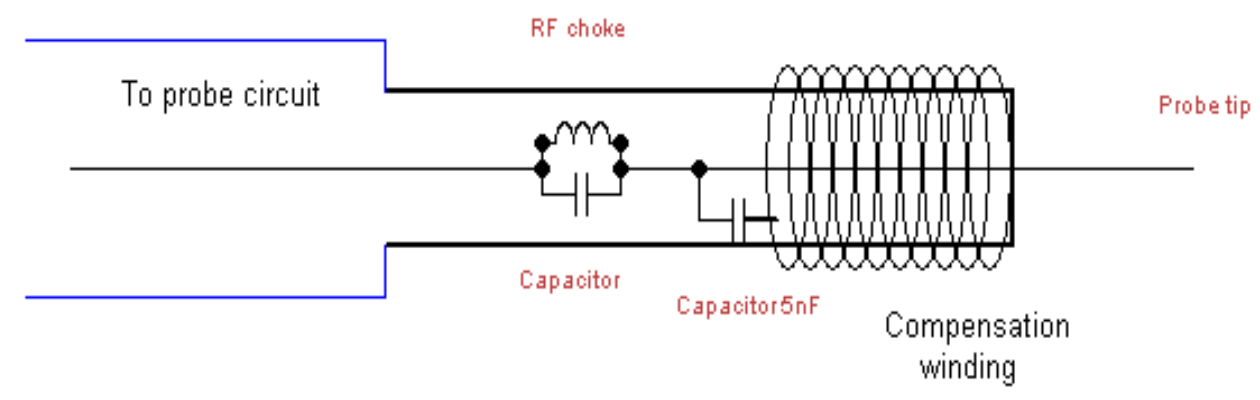

Fig.2: RF Probe construction.

\section{Plasma parameters}

\section{Electron temperature}

Positive ions and electrons are usually approximated to be at the same temperature, but in experimental, these values are different. The electron temperature $T_{e}$ can be found by exploiting the linearity of the electron tardiness region, when viewed on semi $\log$ axes. The electron temperature is representing the reciprocal of the slope of a fitted line to this region. Thus, a conventional definition of the overturned of the slope allows for an explicit representation of the electron temperature in $\mathrm{eV}$ [5].

$$
T_{e}(\mathrm{eV})=\frac{\left(V_{1}-V_{2}\right)}{\ln \left(\frac{i_{1}}{i_{2}}\right)}
$$

where $i_{1}$ and $i_{2}$ are two representative current values residing in this region, and $V_{1}$ and $V_{2}$ are their respective bias voltage values [4].

\section{Electron density}

Obviously the electron density is electron temperature dependent. In general, an assignment of an electron temperature to a non-Maxwellian electron energy distribution is difficult. The electron density $n_{e}$ can be found according to [4]:

$n_{e}=\frac{i_{s e}}{e A_{p}} \sqrt{\frac{2 \pi m}{k T_{e}}}$

where $i_{s e}$ is the electron saturation current, $m$ denotes the mass of the electron and $A_{p}$ is the surface area of the probe [5]:

$$
A_{p}=\frac{D^{2} \pi}{4}+\pi D h
$$

where $\mathrm{D}$ and $\mathrm{h}$ are diameter and length of tip probe respectively [5].

\section{Debye length}

In plasma, electrons are attracted to the neighboring ion and shield its electrostatic field from the rest of the plasma. Similarly, an electron at rest repels other electrons and attracts ions. This effect alters the potential in the neighboring of a charged particle. If plasma had an excess of positive or negative particles, this excess would create an electric field and the electrons will move to cancel the charge [6]. The response of charged particles to reduce the effect of local electric fields is called Debye Shielding and the shielding gives the plasma its quasi-neutrality characteristic [7]. The electric potential developed is mostly near the surface of the particle, extending over a distance $\lambda_{\mathrm{D}}$, called the Debye Length, which is defined by [6]:

$$
\lambda_{D}=\left(\frac{\varepsilon_{o} k T_{e}}{n_{e} e^{2}}\right)^{1 / 2}
$$

Debye length should be very small when compared with the system dimension this first condition for plasma existence: $\lambda_{\mathrm{D}}<<\mathrm{L}$ 
where $\mathrm{L}$ is the dimension system $(\mathrm{cm})$, $\mathrm{e}$ is the electron charge (C), Co permittivity of free space, and $\mathrm{k}$ Boltzmann's constant.

As indicated by the above equation, another plasma parameter related to the Debye length is the number of particles, $\mathrm{N}_{\mathrm{D}}$, in a Debye sphere, that is, in a sphere of radius equal to $\lambda_{\mathrm{D}}$. The solution of Poisson's equation can be obtained only by assuming that the shielding effect is produced by a large number of electrons, or in other words, the shielding effect can occur only if the Debye sphere contains a large number of electrons. Due to the exponential decay of the potential, it can be assumed that the shielding is caused by the electrons in the Debye sphere, whose number is given by [6]:

$$
N_{D}=\frac{4 \pi}{3} n_{e} \lambda_{D}^{3}
$$

The second condition $\mathrm{N}_{\mathrm{D}} \gg 1$ is easily satisfied. The third condition is related to the frequency of collisions in the plasma. If $\tau$ is the mean time between collisions of charged particles with neutral atoms, the product $\omega \tau$ has to be bigger than 1 for the gas to behave like a plasma rather than a neutral gas. The three conditions that have to be satisfied by plasma are therefore given by [6]:

$1-\lambda_{D}<<\mathrm{L} \quad 2-\mathrm{N}_{\mathrm{D}}>>1 \quad 3-\omega \tau>1$

Where $\tau$ is the mean time between collisions of charged particles with neutral atoms (sec) and $\omega_{p}$ is the plasma frequency $(\mathrm{rad} / \mathrm{sec})$ [8].

\section{Experimental setup}

A cylindrical plasma chamber was made from Pyrex class $40 \mathrm{~cm}$ length. The two open ends of plasma chamber were terminated by two stainless steel flange. The, thickness, and diameter of the cylinder are, $5 \mathrm{~mm}$, and $30 \mathrm{~cm}$ respectively. Two planeparallel stainless steel electrodes were used as cathode and anode with $10 \mathrm{~cm}$ diameter. Silicon p-type and n-type is used as a target for sputtering system. RF Plasma is generated in the electrode gap 2, 3, 4, and $5 \mathrm{~cm}$. The discharge was fed with argon at variable flow rate $(100-600) \mathrm{sccm}$. The diameter of the probe tip is $0.2 \mathrm{~mm}$, and the probe holder $3 \mathrm{~mm}$ then increase to $7 \mathrm{~mm}$. The probe length is typically $4 \mathrm{~mm}$. The RF Langmuir probe current-voltage characteristics are obtained by varying the probe voltage between $-150 \mathrm{~V}$ and $150 \mathrm{~V}$ by using DC Power supply to include all the important regions of the curve. A compensated Langmuir probe is used to obtain the I-V characteristic. Use filter inside probe as compensation circuit for measure voltage and current by using DC voltmeter and DC ammeter. The RF probe is cleaned by provides it $200 \mathrm{~V}$ out that glow tip. The RF filter is consist RF chock $10 \mu \mathrm{H}$ connected on parallel with ceramic capacitor 2 pf and by using Eq. (1) we get on filter frequency $4 \mathrm{MHz}$. The pressure values, measured by using Perini Gauge, where pressure values Ranging between 1 to $2 \times 10^{-2}$ mbar by controlling the suction rate of the pumps.

\section{Results and discussion \\ 1. Electron temperature}

The effects of the Argon pressure on the electron temperature of the plasma as shown in Fig.3. The electron temperature drops rapidly as pressure is increased from $\left(7 \times 10^{-2}-1 \times 10^{-1}\right)$ mbar while it becomes relatively constant at higher pressures. At higher pressures there is greater energy transfer between the electrons and the surrounding Argon atoms, which leads to the initial temperature drop. Above $9 \times 10^{-2}$ mbar the limited temperature dependence might be explained by a change in the thermalization process. The electron 
temperature is gradually decreased when increasing the gap distance because the electric field weakens when increasing the distance, so, the current will be less and less energy and hence decrease of electrons temperature as indicated in Fig.4. This result was agree with Chung [2].

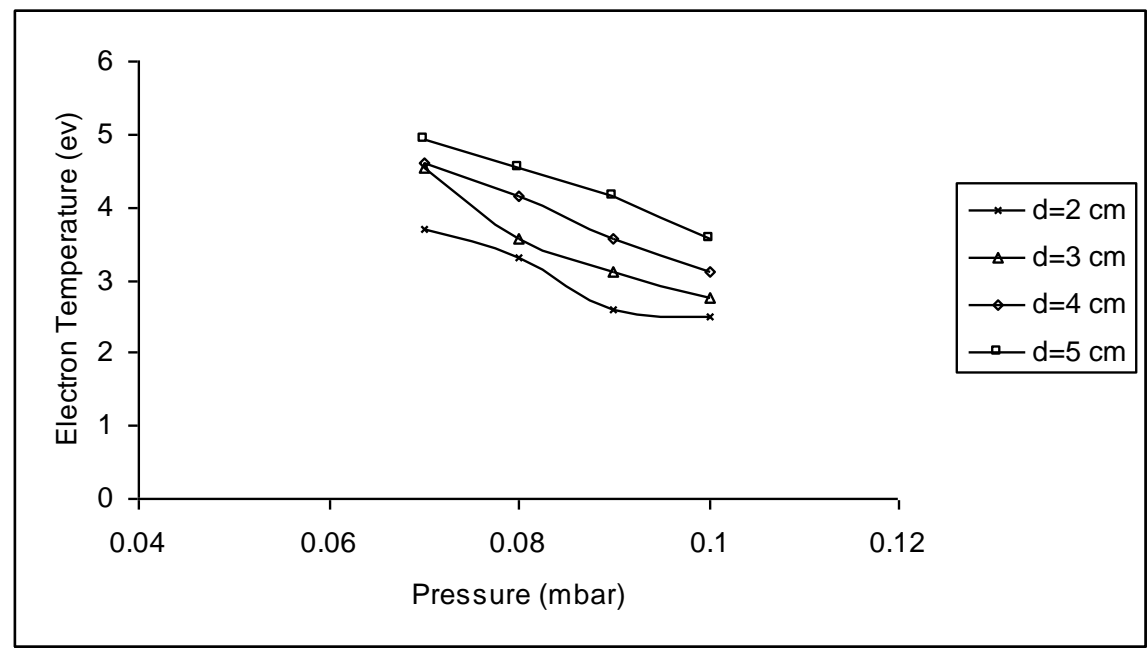

Fig.3: Electron temperatures as a function of plasma pressure for different electrode gaps.

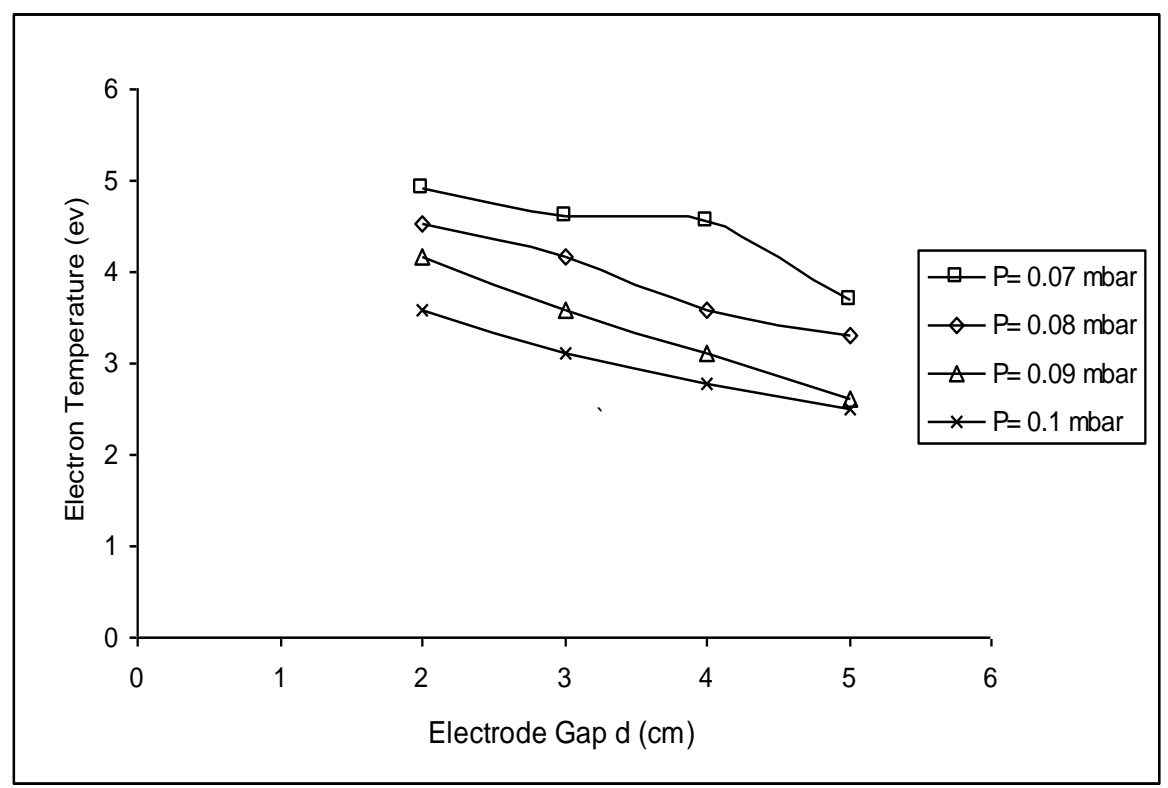

Fig.4: Electron temperatures with electrode gaps at different plasma pressure.

\section{Electron density}

The gas pressure has a major effect on the discharge plasma parameters. Usually, the electron-atom inelastic collision rates are rises by the increase of the gas pressure with a large effect on the plasma density, and electron temperature. When considered a plasma as a fluid, the gas pressure has a major impact on the plasma transport coefficients, which determine the particle and power balance of the plasma. Fig. 5 shows the dependence of electron density on argon pressure. By increase the gas pressure, the 
electron density increased steeply at low gas pressure $<8 \times 10^{-2}$ mbar while it increased little at higher gas pressure.

The electron density drops with increase electrode gap at a constant power, inevitably resulting in the decrease in the electron production rate as shown in Fig. 6 and this result matching with Isola [9].

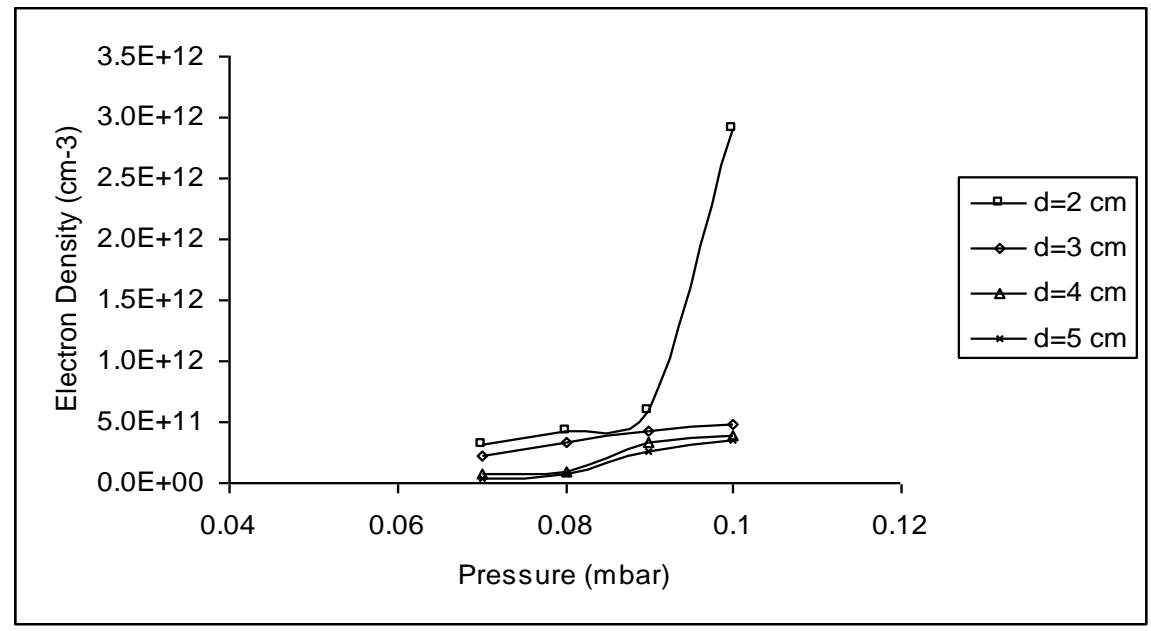

Fig.5: Electron density with plasma pressure at different electrode gap.

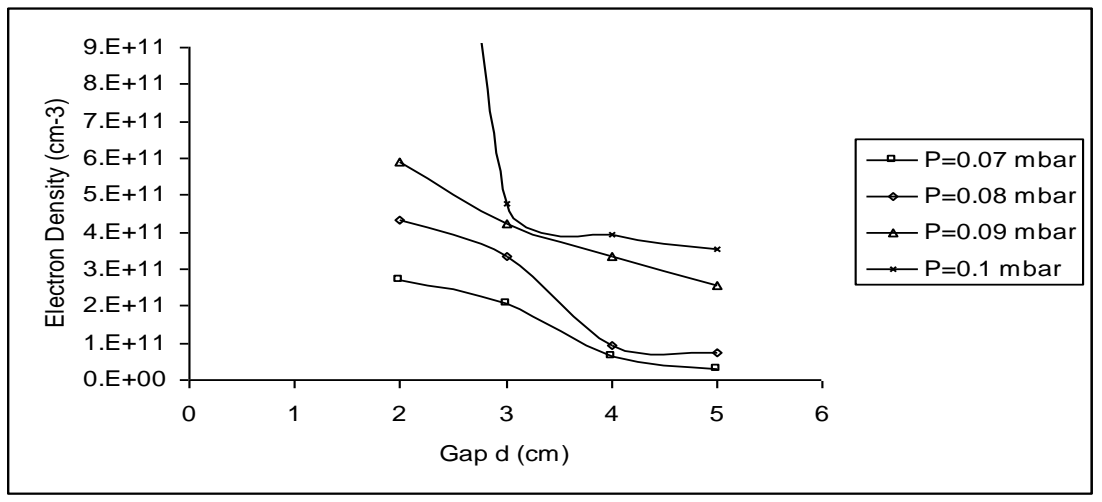

Fig.6: Electron density with electrode gap at different plasma pressure.

\section{Debye length}

From Eq. (5) the Debye length dependent on electron temperature and inversely dependent with electron density. Fig.7 shows that Debye length drops with increases pressure for the same reason of change electron temperature and electron density with electrode gap, Debye length increase with increase electrode gap as shown in Fig.8. This result is matching with Gilchrist [10].

\section{Conclusions}

In this work the $4 \mathrm{MHz} \mathrm{RF}$ argon plasmas are investigated and the technique is based on RF Langmuir probe. The gas pressure has a major effect on the discharge plasma parameters, so that the electron temperature drops slowly as pressure is increased from $\left(7 \times 10^{-2}-1 \times 10^{-1}\right)$ mbar but is relatively drops rapidly at higher pressures, and the electron temperature is gradually decreased when expanding the electrode gap. 


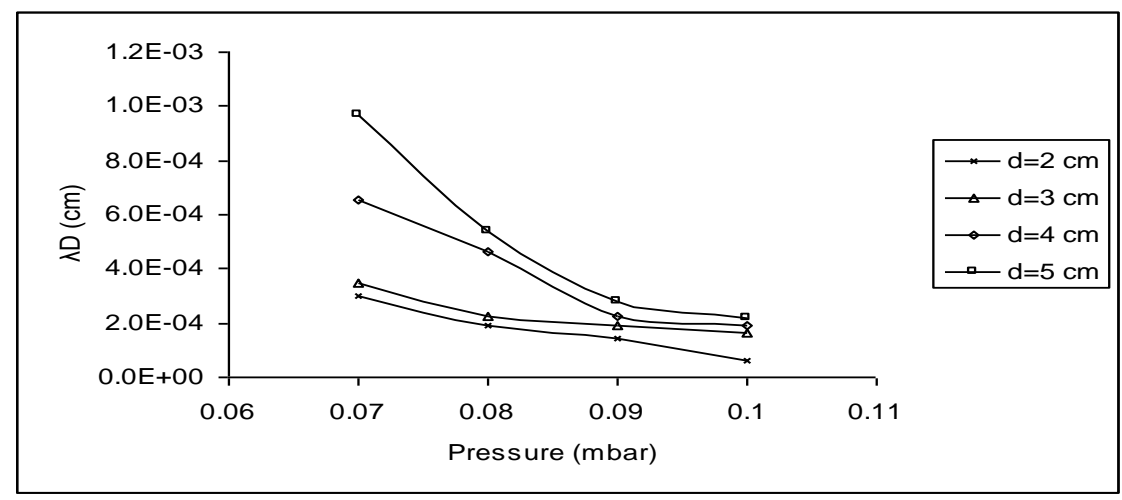

Fig.7: Debye length with plasma pressure at different electrode gap.

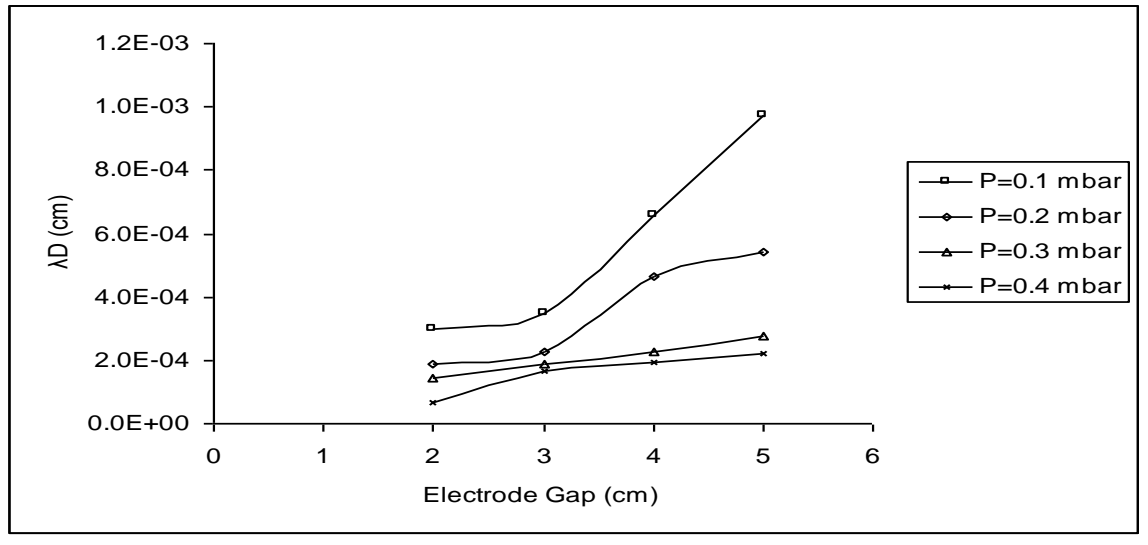

Fig.8: Debye length with electrode gap at different plasma pressure.

Also by increase of the gas pressure, the plasma density rise slightly at low gas pressure $\left(<8 \times 10^{-2}\right.$ mbar $)$ while it drop little at higher gas pressure. But the electron density decreases rapidly with expand distances between electrodes. The changes in electron temperature and electron density with pressure and electrode gap are effect on Debye length.

\section{References}

[1] C.Surdu-Bob, J. Sullivan, S. Ssied, R. Layberry and M. Aflori, Applied Surface Science, 202, 3-4 (2002) 183-198.

[2] T. H. Chung, Hae Ra Kang, Min Keun Bae, J. Physics of Plasmas, 19 (2012) 1.

[3] F. Chen, "Langmuir Probe Diagnostics", J.IEEE-ICOPS, (2003) 1-16.
[4] M. Shaer, A. Soliman, A. Massoud, M. Mobasher, M. Wuttmann, Journal ICPIG, 28, (2007) 1610-1611.

[5] N. Vaghela, "RF (13.56 MHz) Impedance Matching", Huettinger Electronic Inc., (2010) 1.

[6] K. Dobbyn, Dublin City University, M.Sc. Thesis (2000).

[7] B. Doohan, M.Sc Thesis of Science, Dublin City University, (2010) 45.

[8] L. Carroll, "Definition of the Plasma State", Springer-Verlag, (2010) 39-40.

[9] M. Isola, B. Gomez, and V. Guerra, J. of Physics, 43 (2010) 6-8.

[10] E. Gilchrist, G. Bilén, and D. Gallimore, "Current Collection to Long, Thin Probes in a Dense High-speed Flowing Plasma", Michigan Technique Corporation and NASA-MSFC, (2000) 4-5. 